Ann. Génét. Sel. anim., I978, 10 (2), I8I-I89.

\title{
Gènes de coloration du pelage de chèvres Alpines chamoisées et Poitevines
}

\author{
J. J. LAUVERGNE \\ Département de Génétique animale, \\ Centre national de Recherches zootechniques, I.N.R.A. 78350 Jouy-en-Josas
}

\section{Résumé}

Trois allèles au locus $A$ gouti : $A^{b}$ (blaireau) $\mathbf{A}^{t p}$ (noir et feu à ventre clair encore appelé noir et feu poitevin) et a (noir récessif) permettent d'interpréter les ségrégations pour la couleur du manteau observées sur des croisements entre chèvres Alpines chamoisées et chèvres Poitevines au domaine I.N.R.A. de Galles par Avord en 1970-73. La dominance de $A^{b}$ et de $A^{t D}$ sur $a$ est totale : les génotypes $A^{b} a$ et $A^{b} A^{b}$ présentent le phénotype dit " chamoisé " ou "blaireau " avec le corps fauve, le ventre noir, des marques noires sur les pattes et la tête et une raie dorsale noire; les génotypes $A^{t p} A^{t p}$ et $A^{t p} a$ présentent le dessin dit " noir et feu à ventre clair " ou "poitevin": noir avec le ventre clair et des marques claires sur les pattes et la face. Le génotype $A^{b} A^{t p}$ a une expressivité variable : tantôt $A^{b}$ est totalement dominant sur $A^{t p}$ (chez 65 p. Ioo environ des $A^{b} A^{t p}$ ) tantôt on observe un phénotype intermédiaire dit " chamoisé à ventre clair " qui rappelle le phénotype de la Chèvre sauvage.

\section{Introduction}

La chèvre domestique présente une assez large gamme de couleurs dans son pelage mais le déterminisme héréditaire de ces colorations n'est pas totalement élucidé (cf. BERGE, I967; SEARLE, I968).

Pour tenter de combler certaines lacunes, nous avions déjà procédé à l'analyse d'une série de croisements, pour l'étude de la couleur blanche (RICORDEaU et LAUVERGNE, I97I). Dans le présent article nous allons maintenant essayer d'examiner l'hérédité des formes pigmentées observables en France dans les races Poite. vine et Alpine chamoisée, explicitant une première note initiale (LAUUVERGNE, r977). 


\section{Matériel et méthodes}

\section{A. - Origine des données}

Pour une expérimentation de sélection laitière, deux souches avaient été constituées au Domaine expérimental I.N.R.A. de Galles par Avord près de Bourges (Cher): une souche Alpine Chamoisée et une lignée Poitevine sélectionnée après réforme à partir d'une souche Poitevine constituée en $\mathbf{I} 966$.

En I 970 ces deux lignées ont été croisées. On a ensuite procédé à quelques back-cross à partir de sujets qui semblaient intéressants. Les dernières données de coloration ont été relevées en 1973.

\section{B. - Mode de recueil des données}

Tous les reproducteurs utilisés mâles et femelles et tous leurs descendants ont été photographiés dans des conditions identiques d'éclairement et de surface sensible (diapositive Kodakrome prises au flash).

\section{C. - Identification des phénotypes colorés}

Un seul patron coloré est inscrit au standard de la Chèvre Poitevine alors qu'il y en a plusieurs à celui de l'Alpine chamoisée qui peut être chamoisée, mantelée, polychrome ou noire (ITOVIC). Dans la figure I est donné le patron de
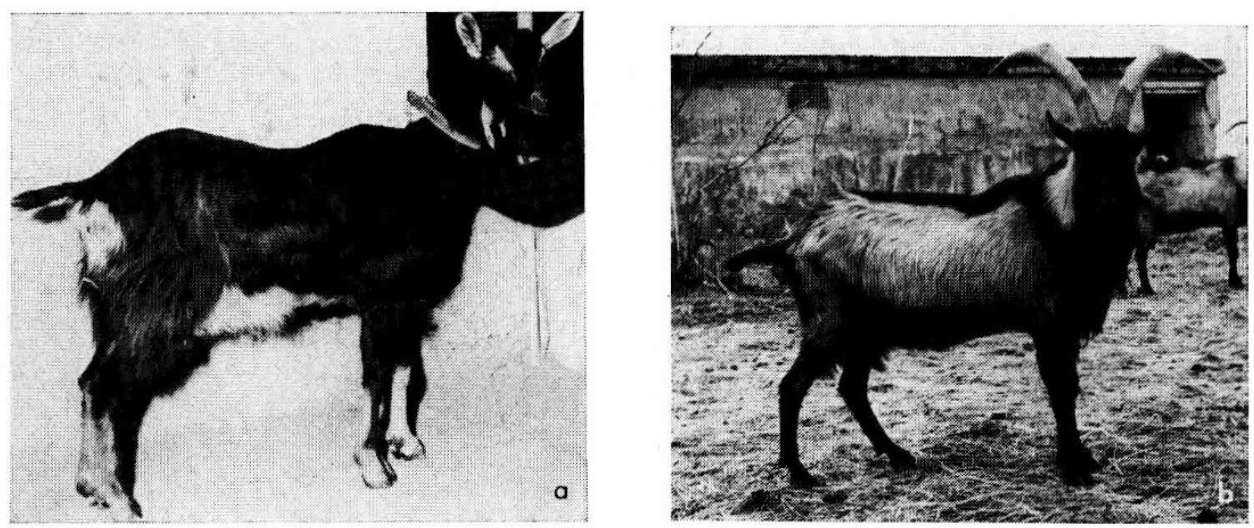

FIG. I. - Phénotypes colorés des races Poitevine et Alpine chamoisée.

a) Le patron " noir et feu à ventre clair " ou " noir et feu poitevin " unique phénotype au standard de la race Poitevine.

b) le patron "chamoisé " ou "blaireau ", le patron le plus courant au sein de la race Alpine chamcisée.

Clichés J. WEBER, C.N.R.Z.

Colour phenotypes in Poitevine and Alpine chamoisé goats

a) "Black and tan light belly" pattern or "poitevin black and tan ", the unique phenotype at the standard of Poitou goat.

b) "chamoisé " or "badger face" pattern, the most frequent colour pattern among the Alpine Chamoisée goat. 
1a Poitevine et le dessin chamoisé de l'Alpine, patron le plus courant dans cette race.

Même si on ne s'occupe pas des panachures blanches plus ou moins irrégulières et assez rares d'ailleurs dans nos données on a identifié au total 7 différents patrons parmi nos lignées de départ et nos croisements. Cinq d'entre eux avaient déjà été décrits par BERGE (I967). Leur définition et leur terminologie sont données dans le tableau I, cependant que les silhouettes sont schématisées dans la figure 2 .
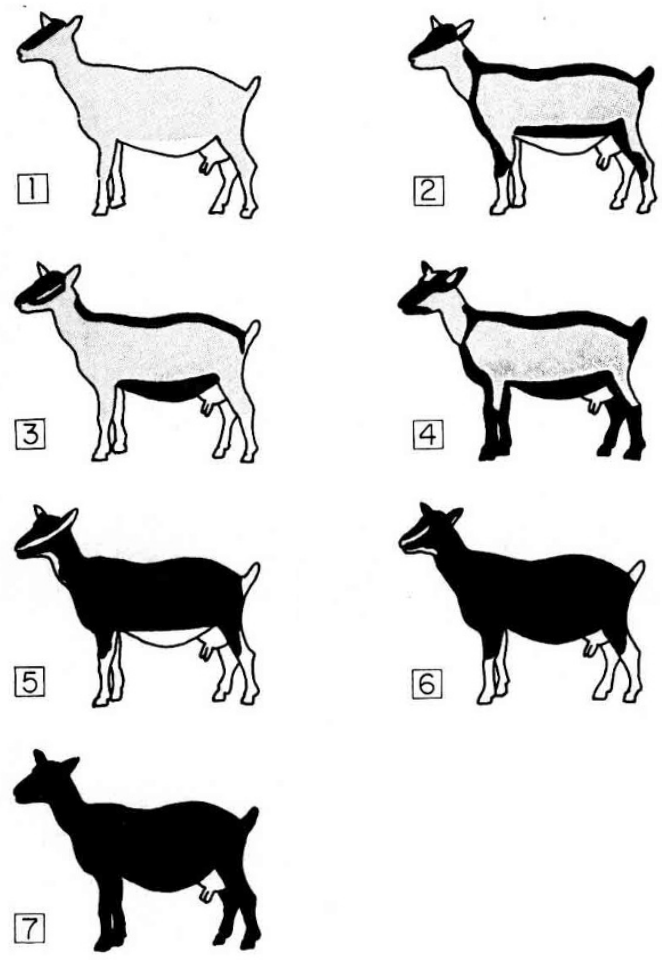

FIG. 2. - Principaux phénotypes rencontrées dans les lignées parentales et en $F_{1}$.

I. Chamoisé clair à ventre clair.

2. Chamoisé à ventre clair.

3. Chamoisé clair.

4. Chamoisé ou blaireau.

5. Noir et feu à ventre clair ou noir et feu poitevin ou poitevine.

6. Noir et feu.

7. Noir.

Main colour phenotypes in parental and $F_{1}$ strains.

I. Light belly, light badger face.

2. Light belly badger face.

3. Light badger face.

4. Badger face.

5. Iight belly black and tan or black and tan Poitou or Poitou.

6. Black and tan.

7. Black. 


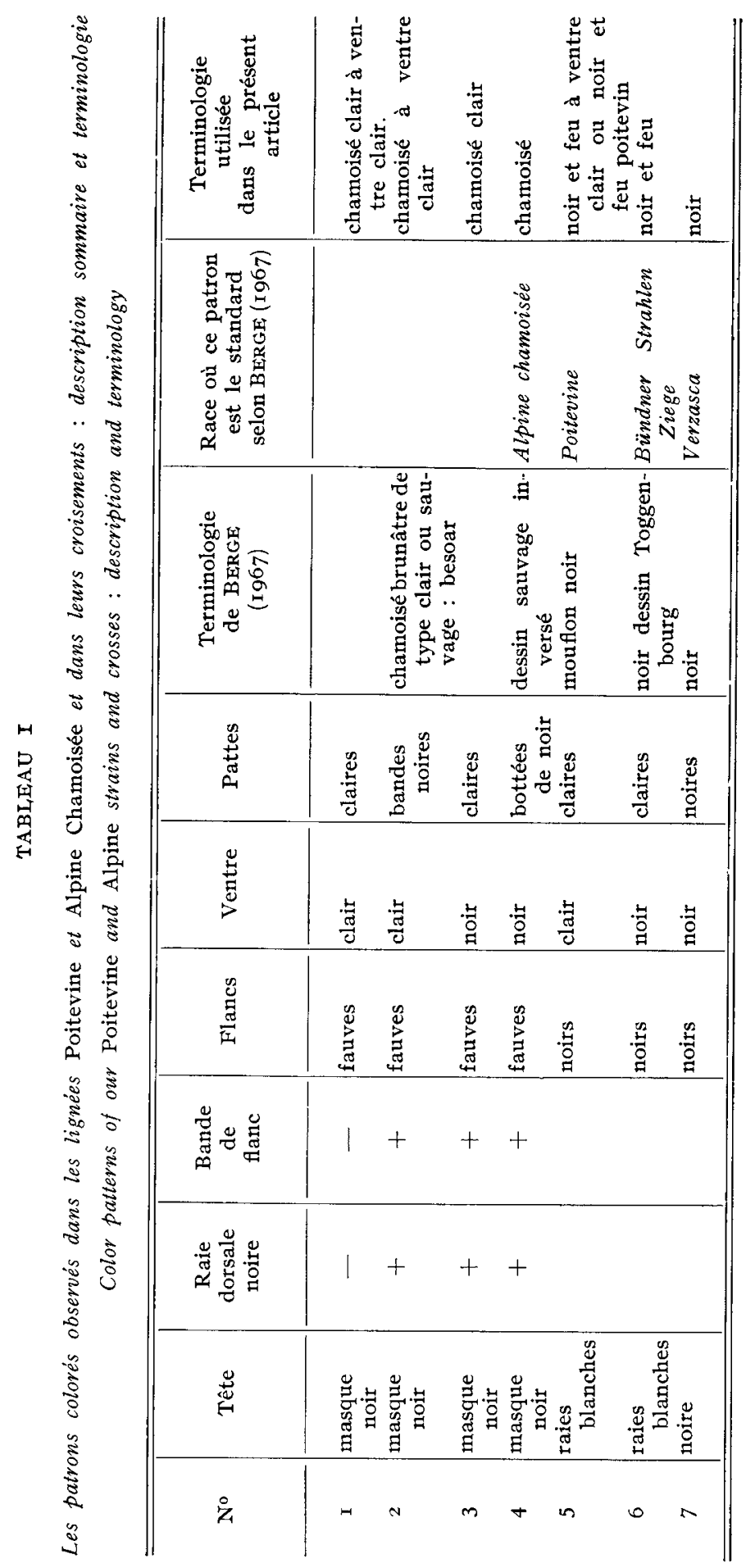




\section{Résultats}

Dans le tableau 2 on a consigné les fréquences des différents phénotypes dans les populations de départ, Poitevine et Alpine chamoisée.

Les résultats des ségrégations observées à l'intérieur des deux lignées, entre les lignées et au cours de croisements supplémentaires sont rassemblés dans le tableau 3 .

TABLEAU 2

Fréquence des différents phénotypes dans les lignées parentales

Frequences of various phenotypes in parental strains

\begin{tabular}{|c|c|c|c|c|c|c|c|c|c|}
\hline & Sexe & $\begin{array}{l}\text { Cha- } \\
\text { moisé } \\
\text { clair } \\
\text { ventre } \\
\text { clair }\end{array}$ & $\begin{array}{l}\text { Cha- } \\
\text { moisé } \\
\text { ventre } \\
\text { clair }\end{array}$ & $\begin{array}{l}\text { Cha- } \\
\text { moisé } \\
\text { clair }\end{array}$ & $\begin{array}{l}\text { Cha- } \\
\text { moisé }\end{array}$ & $\begin{array}{l}\text { Noir } \\
\text { et feu } \\
\text { ventre } \\
\text { clair }\end{array}$ & $\begin{array}{l}\text { Noir } \\
\text { et } \\
\text { feu }\end{array}$ & Noir & $\begin{array}{l}\text { To- } \\
\text { tal }\end{array}$ \\
\hline Lignée Poitevine & 우 & & & & & $6 I$ & I & 7 & 69 \\
\hline $\begin{array}{l}\text { Lignée Alpine } \\
\text { Chamoisée }\end{array}$ & $\begin{array}{l}0 \hat{0} \\
\text { कृष }\end{array}$ & 2 & $\begin{array}{l}\text { I } \\
5\end{array}$ & 4 & $\begin{array}{c}9 \\
43\end{array}$ & & & & $\begin{array}{l}\text { Io } \\
54\end{array}$ \\
\hline
\end{tabular}

\section{Discussion}

\section{A. - Formulation des hypothèses}

Compilant les données disponibles et utilisant les ressources de la génétique comparée SEARLE (I968) proposait un triallélisme au locus Agouti pour expliquer le patron sauvage $(A)$, le noir et feu (black and tan) $\left(a^{t}\right)$ et le noir récessif $(a)$ de la chèvre.

En fait, tout d'abord, à côté du noir (sans doute $a a$ ), nous avons affaire à 2 types de " noir et feu ", l'un avec le ventre clair (le standard coloré de la chèvre Poitevine) l'autre avec le ventre noir (le standard coloré de la Bündner Strahlen Ziege selon BERGE, I967).

Il y aurait peut être donc lieu de postuler l'existence de 2 allèles noir et feu : $A^{t p}\left({ }^{1}\right)$ pour le type " poitevin ", gardant $\mathrm{A}^{t}$ pour le type à ventre noir qui est plus proche des " noir et feu " d'autres espèces.

Le terme de "noir et feu" donné par SEARLE (I968) a été préféré à celui de " mouflon noir " utilisé par BERGE (I967) pour la chèvre suite à une comparaison avec un patron décrit par ce même auteur chez le mouton Scandinave rappelant, en plus foncé celui de l'espèce sauvage : le Mouflon, BERGE (I964). En effet, chez la chèvre, 1'homologie avec le "noir et feu » des autres Mammifères est beaucoup plus 


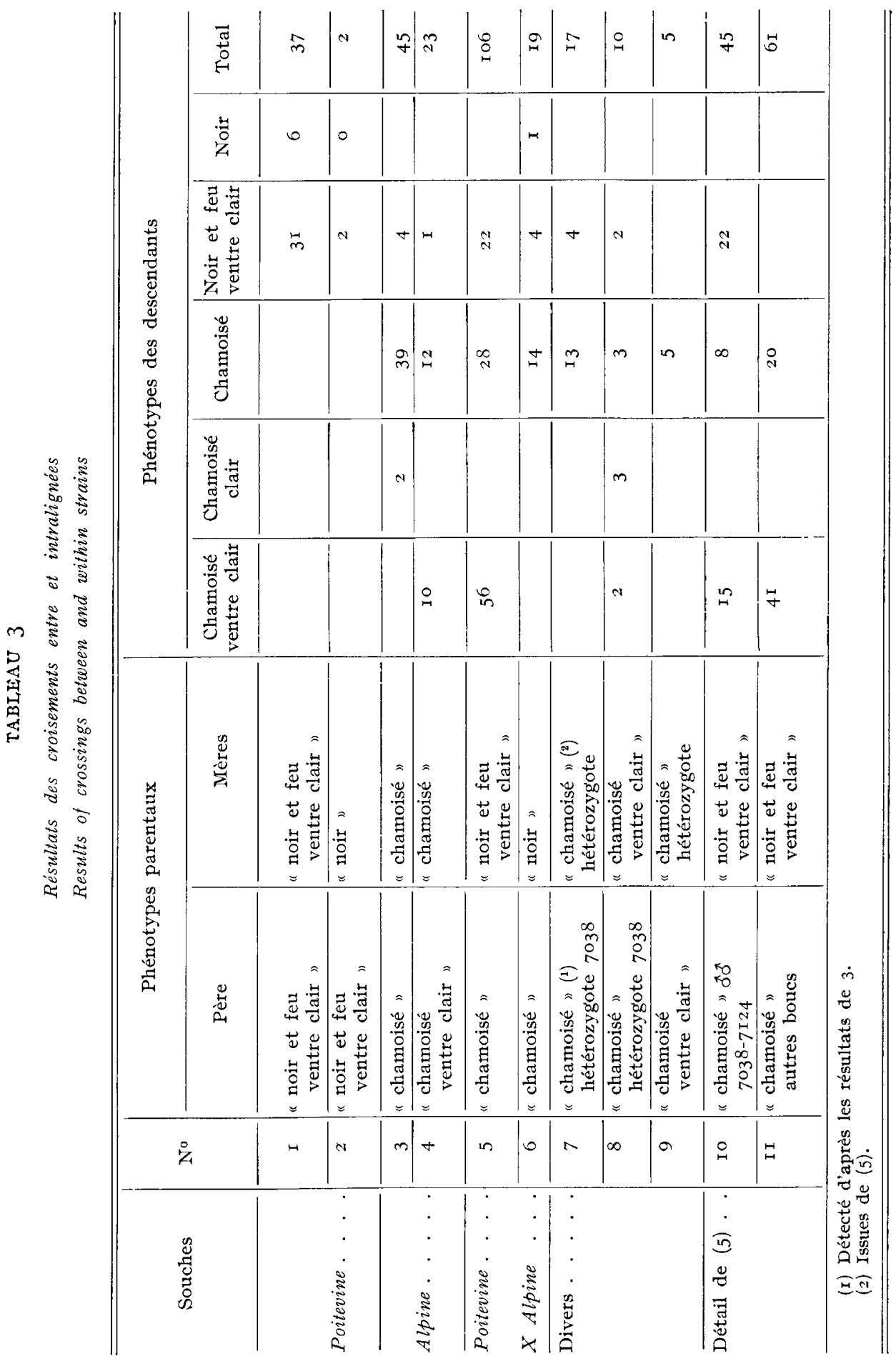


marquée qu'avec le patron sauvage. En particulier, il n'y a pas de poils zonés sur le dos et les flancs qui sont noir uniforme et, parfois, les plages claires, au lieu d'être quasiment blanches sont rouge feu, comme nous 1'avions déjà noté (LAUVERGNE, I970).

Le "chamoisé " (fauve à ventre noir) quant à lui est l'homologue du patron "blaireau " chez le mouton (le "badger face " des auteurs anglo-saxons depuis RoBERTS, I924). Il est déterminé chez le mouton par un autre allèle en Agouti: $A^{b}$ selon Adalsteinsson (I974).

Ainsi nous avons déjà pour 4 des 7 phénotypes du tableau I une interprétation génotypique par un multiallélisme en $A$ gouti: $A^{b}, A^{t p}, A^{t}$, a pour reprendre l'ordre de dominance observée chez le mouton.

$\mathrm{Si}$, dans un premier temps on considère que le " chamoisé clair » ( $\mathrm{n}^{0} 3$, tableau $\mathrm{I}$ ) n'est qu'une variante du " chamoisé " il resterait alors à interpréter le "chamoisé (clair ou non) à ventre clair " (no I et 2 du tableau $\mathrm{I}$ ).

Comme ces phénotypes rappellent le phénotype sauvage (le besoar de BERGE, I967) on peut être tenté d'introduire 1'allèle sauvage $A^{+}$. Toutefois, chez le mouton on a déjà vu qu'un patron un peu semblable pouvait être induit par l'hétérozygote $A^{b} A^{t p}$, ce qui pourrait être aussi le cas chez la chèvre (Adalsternsson, r970).

Ces hypothèses, qui sont les plus simples possibles, tout en tenant compte des données de l'homologie entre loci de coloration des mammifères sont présentées dans le tableau 4.

\section{TABLEAU 4}

Les hypothèses de départ pour la correspondance phénotype génotype

Corresponding phenotypes to genotypes in our basic hypothesis

\begin{tabular}{|c|c|}
\hline Phénotypes & Génotypes \\
\hline $\begin{array}{l}\text { "Noir " } \\
\text { "Noir et feu " } \\
\text { "Noir et feu à ventre clair " (" noir et feu poi- } \\
\text { tevin ") } \\
\text { "Chamoisé " } \\
\text { "Chamoisé ventre clair" }\end{array}$ & $\begin{array}{l}a a \\
A^{t} A^{t}, A^{t} a \\
A^{t p} A^{t p}, A^{t p} A^{t}, A^{t p} a \\
A^{b} A^{b}, A^{b} A^{t p}, A^{b} A^{t}, A^{b} a \\
A^{+} A^{+}, A^{+} A^{b}, A^{+} A^{t p}, A^{+} A^{t}, A+a \\
\quad \text { ou } A^{b} A^{t p}\end{array}$ \\
\hline
\end{tabular}

\section{B. - Test des hypothèses}

Comme il n'apparaît ni " chamoisé clair à ventre clair », ni " noir et feu " dans nos croisements et que les " chamoisé clair " sont rares (tabl. 3) la discussion sera limitée à l'interprétation de 4 des cinq phénotypes énumérés dans le tableau 4 : " noir ", " noir et feu à ventre clair ", ou " noir et feu " poitevin, "chamoisé " et " chamoisé à ventre clair " avec les allèles suivants en $A$ gouti: $a, A^{t p}, A^{b}$ et $A^{t}$.

(I) A partir de maintenant on notera les allèles en A gouti avec le symbole du locus invariable et, en exposant, une lettre ou un signe consacrés par la tradition ou bien la première lettre du nom de l'allèle, à l'exception du terme le plus récessif $a$ donnant le noir. 
Les variations intra lignée du tableau 2 montrent que celles-ci ne sont pas homozygotes, ce qui s'interprète parfaitement avec les allèles $A^{t p}$ et $a$ pour la lignée Poitevine (croisements $\mathrm{n}^{\circ} \mathrm{I}$ et $2 \mathrm{du}$ tableau 3). La fréquence de $a$ dans cette lignée est alors de l'ordre de 0,3 à partir des comptages du tableau 2 et de 0,65 à partir du croisement $n^{0}$ I, tableau 3, si l'on suppose la panmixie.

Au sein de la lignée Alpine chamoisée les ségrégations en 3 (tab1. 3) s'expliquent par la présence chez les animaux "chamoisés " de $A^{b}$ dominant sur $A^{t p}$ et presque toujours à l'état homozygote cependant que les résultats des accouplements 4 s'interpréteraient en disant que les "chamoisés à ventre clair " sont de formule $A^{+} A^{b}, A^{+}$étant dominant sur $A^{b}$.

Toutefois cette interprétation, qui fait intervenir $A^{+}$, est mise en échec par les résultats du croisement entre lignées $n^{0} 5$ du tableau 3 où les " chamoisés à ventre blanc " sont produits en abondance lors d'accouplements " chamoisé " $\left(A^{b} A^{b}\right.$ ou $\left.A^{b} A^{t p}\right)$ par " noir et feu poitevin " $\left(A^{t p} A^{t p}\right.$ ou $\left.A^{t p} a\right)$, étant donc manifestement de formule $A^{b} A^{t p}$. Il est donc plus avisé d'admettre cette seconde interprétation d'une double expressivité du génotype $A^{b} A^{t p}$ : soit en dominance simple de $A^{b}$ sur $A^{t p}$ soit en interaction pour produire un phénotype intermédiaire entre "chamoisé " et " noir et feu poitevin".

L'estimation rigoureuse de l'expressivité de la forme intermédiaire "chamoisé à ventre clair " parmi les hétérozygotes $A^{b} A^{t p}$ exige des calculs compliqués car il faut tenir compte de diverses fréquences géniques. En première approximation on peut cependant la situer aux alentours de 0,65 lors des croisements entre lignées (Io et II du tabl. 3) et de 0,45 en race Alpine chamoisée pure ( ${ }^{\circ} 4$ du tabl. 3).

Au cas où cette différence serait significative on pourrait l'expliquer par une sélection en faveur du type "chamoisé " plutôt que "chamoisé à ventre clair " au sein de la race Alpine. Bien que les éleveurs fassent souvent mal la différence entre "chamoisé " et " chamoisé à ventre clair ", ils choisissent cependant, au moins pour les mâles, des animaux dont la coloration ventrale noire est bien développée.

En fait le problème de la " mimique " du phénotype sauvage par le phénotype induit par $A^{b} A^{t p}$ pourrait peut-être être résolu par un examen plus attentif de ces deux patrons, encore faut-il disposer de ces deux génotypes.

Rę̧u pour publication en juillet 1978 .

\section{Remerciements}

Ce travail n'aurait pas vu le jour sans l'aide de l'équipe de Galle alors sous la direction scientifique de G. RICORdeAu. On voudrait citer en particulier P. GurLimin.

Le manuscrit a été relu par le Dr SEARLE, Medical Research Council, Harwell, Didcot, Angleterre dont les conseils ont été fort profitables.

\section{Summary}

Coat color genes in crosses between Alpine Chamoisée and Poitou goats

The segregations for coat colour in a crossing between Alpine Chamoisée and Poitou goats at the I.N.R.A. estate of Galles nearby Bourges (France) may be interpretated with three alleles. in $A$ gouti locus : $A^{b}$ (badger face), $A^{t p}$ (black and tan Poitou) and $a$ (recessive black). The dominancy of $A^{b}$ and of $A^{t p}$ upon $a$ is total: $A^{b} a$ and $A^{b} A^{b}$ genotypes are "badger face" with a 
fawn body, black belly and black marks on legs and head plus a black dorsal stripe; the $A^{t D} A^{t D}$ and $A^{t p_{a}}$ genotypes give a black and tan phenotype, black with light belly and white stripes on face and legs. In the $A^{b} A^{t p}$ genotype $A^{b}$ is dominant upon $A^{t p}$ with a penetrance of $65 \mathrm{p}$. 100, the remaining $45 \mathrm{p}$. I $A 0$ of heterozygotes showing an intermediary pattern called "light belly badger face " which is similar with the phenotype of the wild goat.

\section{Références bibliographiques}

Adalsteinsson S., r970. Colour inheritance in Icelandic Sheep and relation between colour, fertility and fertilization. J. agr. res. Icel., 2, 3-135.

Adalsteinsson S., 1974. Colour inheritance in farm animals and its application in selection. Irst World Congr. Genet. appl. Livest. Prod., 1, 29-37.

BERGE S., 1964. Sheep colour Genetics (Norvégien) (Engl. summary). Meld. Norg. Landbrukshøgsk., 43 (6), $32 \mathrm{p}$.

Berge S., I967. A propos de la couleur des chèvres. Rev. Élév., 22 (4), III-II5, (6/7) I2I-I 24, (Io) II 7-I II.

ITOVIC (sans date). Les races caprines françaises. Coframinex, Paris, 2 tracts non paginés.

LAUVERGNe J. J., I97o. Mise en évidence de l'existence du phénotype noir et feu dans deux nouvelles espèces de Mammifères. Revue roum. Biol., 15, II3-II8.

LaUvergne J. J., 1977. New alleles at the Agouti locus $A$ in domestic Ruminants. Proc. Xth Pigment Cell Conf. Boston, oct. r977 (Abstr).

Ricordeau G., LAUvergne J. J., I97I. Déterminisme héréditaire de la couleur blanche de la chèvre Saanen. Ann. Génét. Sél. anim., 3, 425-432.

ROBERTS, J. A. F., I924. Colour inheritance in sheep I. Black colour and badger-face pattern in Welsh Mountains sheep. J. Genet., 14, 367-374.

SEARLE A. G., 1968. Comparative Genetics of Coat Colour in Mammals. Logos Press, Academic Press, London, New-York, 195. 